\title{
Estado del arte: el aprendizaje reflexivo en la formación inicial con los estudiantes de Prácticas de Formación Profesional en la carrera de Inglés
}

\author{
M.Sc. Skarlet Romero Espinoza \\ Docente investigadora \\ Máster en Metodología y Didáctica para la Educación Superior \\ UNAN-Managua, FAREM-Carazo \\ skarlet.romero.espinoza@hotmail.com
}

Fecha de recepción: 27 de octubre, 2019 / Fecha de aceptación: 21 de abril, 2020

https://doi.org/10.5377/torreon.v9i25.9853

Palabras clave: aprendizaje reflexivo, formación inicial, practicas, formación profesional.

\section{RESUMEN}

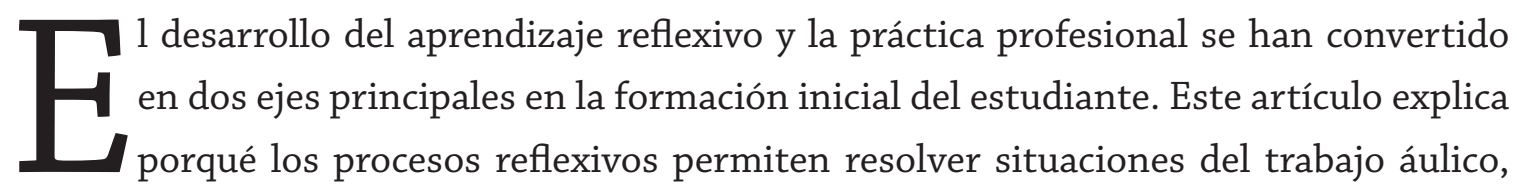
que es construido y transformado durante las prácticas de formación profesional relacionando el aprendizaje reflexivo, formación inicial del estudiante con el rol de las prácticas pedagógicas (como profesor en formación, tutor o guía).

\section{INTRODUCCIÓN}

El sector educativo se ha interesado por la temática de la práctica reflexiva pero resulta todo un desafío su implementación en las prácticas profesionales. Las prácticas profesionales conforman una parte importante en la formación profesional, debido a que ayuda al desarrollo 
de la inserción al trabajo áulico, están ligadas a un conjunto instrumental de actividades y a saberes concretos en el manejo de la dinámica de una clase.

Porlotantolas prácticas profesionales constituyen una entidad coherenteinterdependiente dentro del currículum de formación docente, permiten comunicar al practicante con acciones institucionalizadas dentro y fuera del ámbito universitario, producidas en variedad de escenarios en los cuales observa, interviene, reflexiona, reconstruye y valora realidades en su complejidad; hecho que precisa de una serie de herramientas conceptuales, procedimentales y actitudinales con la intención de ir construyendo su identidad como docente.

Por consiguiente se formula la desvinculación entre la teoría y la práctica en la formación inicial docente quienes la expresan con mayor convicción, aún sigue siendo privilegiar la formación teórica. La aplicación del modelo reflexivo es la vía que hace posible comprender la vinculación entre la teoría y la práctica y en esa estrecha relación se genera conocimiento teórico -práctico, la formación en la reflexión hace un análisis de los conocimientos teóricos- prácticos hacia la revisión de las propias concepciones acerca de la educación, sus propias experiencias y la relación con la puesta en práctica. Se pretende formar docentes críticos, responsables, reflexivos, éticos que sean capaces de generar espacios pedagógicos.

En relación con lo anterior se propone la implementación del aprendizaje del modelo reflexivo este ayudará a mejorar las prácticas haciendo uso de las estrategias reflexivas tales como los diarios de clase y los portfolios donde escribirán sus reflexiones acerca de sus propias experiencias en el entorno educativo facilitando el proceso de enseñanza aprendizaje.

Stenhouse (1997) define que durante el curso de los estudios de una carrera el puente entre lo teórico y lo práctico lo constituye el currículo. De modo que, los estudiantes al desplazarse por los distintos componentes curriculares, y en este caso, el componente de prácticas profesionales, tienen la posibilidad de abordar teoría y práctica para nutrir la una de la otra y en ese intercambio entender el carácter transformador y transformable del proceso educativo, siempre y cuando lo aborden desde la reflexión.

Detengámonos un poco en la idea de la reflexión desde la práctica, que permita a los estudiantes reflexionar desde sus prácticas en el aula, al participar en la realidad educativa, para comprender su significado y alcance. Por tanto es necesario tomar la decisión de formar al estudiantado en la práctica reflexiva empoderándolos del trabajo en el salón de clase.

Presentar el estado del arte referido a la acometida existente que se hace del tema, el aprendizaje reflexivo en la formación inicial con los estudiantes de formación profesional, es el objetivo principal de este artículo, al encontrar esos aportes teóricos que llevados a la práctica pueden aportar al trabajo áulico en la formación inicial del profesorado. 
Se consideran algunos fundamentos teóricos, así como algunos trabajos investigativos relacionados con los temas en cuestión, desarrollados en algunos países de América Latina como Colombia, Chile, México. Se presentan en este trabajo una sección acerca de los conceptos de reflexión, una segunda sección dedicada a literatura, donde se aborda la diferencia conceptual entre las Prácticas Profesionales Docentes y las prácticas universitarias, una tercera sección, el lugar marginal que ocupan las Prácticas Profesionales dentro del escenario de la formación docente seguido de algunos estudios relacionados y conclusiones.

\section{DESARROLLO}

\section{El aprendizaje reflexivo en la formación inicial}

El aprendizaje reflexivo es un modelo de formación que se fundamenta en las teorías socioculturales del aprendizaje humano. Allwright (2005), Alsina (2007), Brockbank y McGill (2002), Esteve (2004) y Esteve, Melief y Alsina (2010), señalan que algunos rasgos representativos del aprendizaje reflexivo, o aprender a partir de la práctica, son la interacción, la reflexión y el contraste para poder construir y reconstruir conocimiento.

De acuerdo con Dewey (1989) el profesor reflexivo se caracteriza por poseer una mente abierta y ser sincero, se pregunta por las razones que determinan sus acciones y las consecuencias de las mismas, haciéndose responsable por los resultados, no se conforma con el logro de los objetivos sino que cuestiona si los resultados son satisfactorios, y la reflexión la realiza antes, durante y después de la acción.

Como señala el autor los docentes tenemos que ser honestos, leales, responsables con nuestra labor docente, ya que nuestras acciones inciden en los estudiantes.

Zeichner (1993) define que la reflexión desde la práctica, se sustenta en dos principios básicos. El primero reconoce la condición profesional de los educadores y su papel protagónico en el proceso de enseñanza y aprendizaje; el segundo, establece la capacidad de los docentes para generar saber pedagógico. Desde esta perspectiva, el conocimiento de los profesores es útil y les sirve no solo para desarrollar saber práctico sino para investigar su práctica y producir saber teórico, no tienen que limitarse a aplicar ideas creadas por otros.

Cabe detallar que los estudiantes reflexionen acerca de sus propias prácticas educativas sería convincente que ellos compartan sus experiencias con otros compañeros para valorar su aprendizaje.

Villar (1999) expresa también su visión del profesor reflexivo enfatizando en los aspectos afectivos, morales y sociales de su práctica: Llegar a ser un profesor reflexivo significa e implica algo más que la probidad intelectual de utilizar procesos cognoscitivos de análisis interno de los 
fenómenos curriculares y educativos. Incluye, además, la realización de actividades afectivas y morales en las aulas de los centros escolares, en la comunidad escolar y en las asociaciones sociopolíticas. (p. 22).

Liston y Zeichner (1997) afirma una propuesta de enseñanza reflexiva, organizada en cinco componentes:

- La enseñanza. Actividad en la cual el alumno practicante entra en contacto con la realidad y se va haciendo responsable de la clase; en ese espacio se hace consciente de los supuestos implícitos del currículum, los descubre y articula, y a su vez, efectúa propuestas y ajustes acordes a las necesidades del contexto áulico.

- La investigación. Posibilita a los alumnos en Prácticas la ubicación en los contextos socio-históricos, además, desarrollar capacidades para investigar sobre su propio trabajo y adquirir conocimientos acerca de la cultura escolar. A partir de este análisis proponen la elaboración de proyectos de investigación-acción, estudios etnográficos y proyectos de análisis curricular. Se trata, entonces, de un trabajo colaborativo entre profesores tutores y asesores de Prácticas, así como del apoyo entre pares.

- Los seminarios. Estrategias vinculadas con la investigación, se planifican conjuntamente y se centran en diversas cuestiones como currículum oculto, aprendizaje cooperativo, evaluación, procesos de enseñar y aprender. Surgen de las experiencias de los practicantes en las escuelas, también de lecturas sobre vivencias escritas por profesores y estudiantes de semestres anteriores con la intención de construir diversas perspectivas sobre ciertas problemáticas educativas.

- Los diarios de prácticas. Instrumento cualitativo donde los estudiantes reflejan sus impresiones, reflexiones y su evolución como profesores; constituyen un medio muy importante para generar procesos de reflexión.

- Las tutorías. Proceso que enfatiza las condiciones sociales de los grupos de estudiantes en Prácticas y en sus oportunidades de aprendizaje, estableciendo encuentros previos a las observaciones de clase, acompañamiento y posteriores entrevistas para valorar y evaluar los momentos cruciales de las experiencias didáctico-pedagógicas ejecutadas.

Cabe mencionar quela reflexión y la crítica tendrán la obligación de mejorar las condiciones sociales, políticas y culturales de las sociedades, con base en la participación activa y solidaria encaminada a lograr procesos transformadores e innovadores. Esto será un gran desafío para la educación.

Perrenoud (2004) afirma que en las sociedades actuales ocurren transformaciones rápidamente; las tecnologías, la comunicación se modifican constantemente, se vive rodeado de 
paradojas, por tanto, al educador incumbe prepararse para una práctica reflexiva, estimuladora de la innovación y la cooperación. De este modo, las competencias reflexivas y críticas deben trabajarse desde la formación inicial e ir conformando esquemas mentales con los cuales el estudiante en el futuro cercano encontrará asidero para interpretar e intervenir la realidad educativa.

Por lo tanto el docente tiene la responsabilidad de prepararse para una práctica reflexiva para poder preparar a los estudiantes en su formación inicial, es necesario que los maestros constantemente se preparen al cambio del día a día para mejorar los escenarios pedagógicos e intervenir en la realidad educativa.

Schön (1992) “Aboga por un proceso para desarrollar la reflexión en la acción, comenzando con proveer a los sujetos del entrenamiento técnico, progresando en apoyarlos a pensar como profesionales y, finalmente, permitirles desarrollar nuevas formas de entender la acción".

Donald Schön, (1992) por su parte, destaca el carácter social de los conocimientos implícitos de los docentes a través de la noción de "prácticum". Desde el punto de vista constructivista, señala, nuestras emociones, apreciaciones y creencias tienen sus raíces en los mundos que nosotros mismos formamos y que terminamos por acceder como realidad. La práctica se aprende dice Shön a través de la inclusión en un determinado prácticum. Cuando alguien aprende una práctica, se inicia en la comunidad de prácticos y del mundo de la práctica que estos habitan. Aprende sus percepciones, apreciaciones, limitaciones, lenguajes.

Respecto de dicha práctica reflexiva, la investigación ha acusado un incremento importante en los últimos años. A los trabajos de Villar (1999); Korthagen (1996); Stenhouse (1997); Perrenoud (1996); Zeichner (1993); Liston y Zeichner (1997); Diker y Terigi (1997); Tardif (1997) ; Zabalza (1998) ; Carr y Kemis (1988) que han abordado la investigación de las prácticas reflexivas focalizando su atención especialmente en los modelos y procesos de desarrollo profesional docente y formación de profesores, se suman hoy los de Allwrigh (2005); Brockbank y Mcgill (2002); Sayago (2006); Alsina (2007); Perrenoud (2004); Lee (2005); Chacon (2003); Domingo (2013)); Catellanos (2017) entre muchos otros, la mayor parte de ellos vinculados al debate en torno a la construcción del conocimiento didáctico y profesional.

Todos los trabajos se remontan o tienen como referencia obligada los aportes de dos autores que surgen como los pilares del enfoque: John Dewey y Donald Schön. Los aportes de la investigación apuntan de manera predominante a establecer distinciones entre diversos tipos, contenido y niveles de reflexión, los procesos a través de los cuales se adquiere y desarrolla esta así llamada "metacompetencia reflexiva", y a enunciar su vinculación con ciertas condiciones sociales del ejercicio profesional. La idea, señala Korthagen (1996), de que el profesor puede aprender a someter su propio comportamiento al análisis crítico y hacerse responsable de sus 
acciones, encontrando el gran filtro necesario para una especie de enseñanza que trasciende el simple entrenamiento y el uso de competencias específicas de comportamiento, la toma de decisiones sistemáticas y racionales, está vinculada al corazón de la profesionalización. Esto explica "no sólo la popularidad de la reflexión sino también la forma que el término ha sido interpretado por varios autores" (p. 317).

De acuerdo con esto Korthagen (2001) expresa: "para diseñar las fases de la actividad formativa, partimos del ciclo reflexivo denominado ALACT, a partir de las iniciales de los términos ingleses Action, Looking back to the action, Awareness of essential aspects, Creating alternative methods of action and Trial".

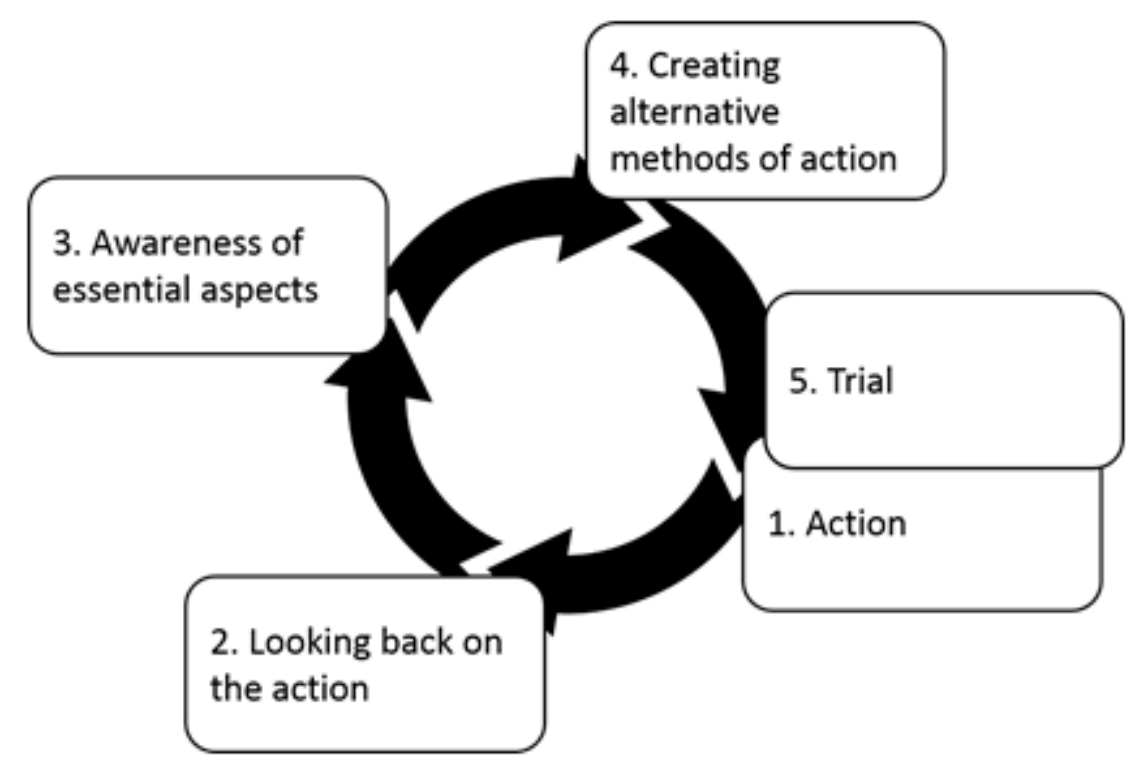

Figura 1. Proceso formativo para promover el ciclo reflexivo Fuente: (Korthagen, 2001)

La figura 1 describe este proceso cíclico en cinco fases: 1. Acción o experiencia (A); 2. Mirando hacia atrás en la acción (L); 3. Conocimiento de un aspecto esencial (a); 4. Creando métodos de acción alternativos $(C) ; 5$. Comprobar en una nueva situación $(\mathrm{T})$ e iniciar un nuevo ciclo, esto se orienta bajo el modelo reflexivo ALACT.

Por lo tanto es necesario que los estudiantes conozcan las fases del ciclo reflexivo para que desarrollen nuevas formas de acción en el entorno educativo brindándole acompañamiento antes, durante y al final de las prácticas.

\section{La diferencia conceptual entre las Prácticas Profesionales Docentes y las prácticas}

En las Prácticas Profesionales, también coinciden concepciones de modelos de enseñanza, aprendizaje, tradiciones de formación docente, modalidades de gestión, administración de instituciones escolares y las características propias del contexto sociocultural. En esta representación, abren un abanico de posibilidades al estudiante, quien analiza críticamente 
su actuación de aprendiz y de enseñante, a la vez tiene oportunidad de discutir, contrastar y reconstruir la experiencia docente asumiéndola como objeto de análisis.

Las Prácticas conducen a acciones integrales directamente en la conformación de la profesionalidad, apuntan a reafirmar los fines y propósitos establecidos en el currículo, por tanto forman parte de un terreno oportuno en el tratamiento de problemas a ser estudiados y reflexionados, cuya orientación sea mejorar el perfil y el desempeño profesional de quien se forma como docente.

De otra parte, la condición de Profesional lleva a considerar ciertos rasgos característicos. Según autores como Carr y Kemmis (1988) y Pérez (1993), se resumen así: "Cuando se alude a la Práctica como una acción Profesional se presume que en la base de los sujetos en formación existe alguna idea sobre la enseñanza en materia de métodos, junto con nociones acerca de la procedencia del conocimiento. Esta acción está sostenida en un cuerpo teórico de conocimientos y requiere de un período de formación académica."

La definición de una Práctica como Profesional trasciende la adquisición de habilidades y destrezas o competencias prácticas, es adquirir valores, intereses y actitudes que caracterizan la profesión.

Concebir la Práctica como Profesional, es a su vez aceptarla como el Eje de la formación docente. En tal caso, los futuros docentes deben disponer del conocimiento necesario para enseñar y poseer competencias y habilidades, analizar su práctica y estar conscientes de las implicaciones ético-valorativas de la enseñanza.

Las ideas anteriores explican los rasgos de las prácticas, como cada aprendiz interpreta la idea de la formación profesional y el concepto de práctica, dado que los profesores deben poseer conocimientos para enseñar nuevas metodologías y habilidades para que el estudiante desarrolle valores -éticos de la enseñanza.

\section{El lugar marginal que ocupan las Prácticas Profesionales dentro del escenario de la formación docente.}

En el contexto universitario las Prácticas Profesionales ocupan un lugar marginal en el campo de la discusión teórica, si se quiere, se encuentran subestimadas en cuanto a la posición que ocupan dentro del ámbito formativo. Por lo tanto se ha caído en el error de situarlas en una perspectiva única, exclusivista operando aisladamente, separada de toda mediación social con los diferentes contextos culturales y conexiones multidisciplinares. Generalmente las prácticas son períodos cortos en los cuales el estudiante facilita y aplica conocimientos previamente consensuados proporcionándole una aceptable actuación dentro del aula de clase. Esta idea 
atribuye a las prácticas en experiencias sostenidas con comportamientos reproductores de esquemas y rutinas docentes.

En relación con este punto, es oportuno destacar el criterio de Diker y Terigi (1997, p. 224). Cuando afirman "la Práctica debe ser el hilo rojo, los objetivos sobre los cuales se trabaja constantemente". De igual manera, con criterios como el de Zabalza (1998) en cuyas palabras las Prácticas deberían convertirse en una pieza importante dentro de la formación inicial, toda vez que ellas inician el proceso de acercamiento a situaciones reales de enseñanza y aprendizaje desde varios propósitos a la vez: como punto de referencia y contraste, aplicación y revisión de conocimientos teóricos ofrecidos en las distintas disciplinas del plan de estudio y para reflexionar sobre ellos. Otro de los criterios es el de Schön (1995, p. 8) quien considera que la Práctica Profesional es la "competencia de una unidad de prácticos que comparten [...] las tradiciones de una profesión, es decir, convenciones de acción, lenguaje y medios, repertorios, sistema de valoración, limitaciones, ejemplos, conocimiento sistemático y sus patrones de conocimiento en la acción."

Finalmente, la reflexión crítica, sostiene que se investiga y reflexiona con el fin de transformar la práctica, interpelando las condiciones sociales de la experiencia docente, concibiéndola al igual que las dos tradiciones anteriores -como una herramienta al servicio del mejoramiento de la práctica, pero desde un abordaje social y contextual que enfatiza en la transformación (Zeichner, 1993).

Por su parte, Liston y Zeichner (1997), asignan a las Prácticas una función de aprendizaje constante en la enseñanza que excede la demostración y aplicación de conocimientos y técnicas adquiridos por el estudiante de educación durante el trayecto de formación. En esta concepción, se trata de priorizar la autorreflexión y valoración por las acciones realizadas en ambientes de respeto mutuo y establecimiento de relaciones de colaboración entre profesores de las escuelas y los practicantes. En definitiva, son experiencias estimuladoras hacia el conocimiento de sí mismo y la capacidad para aprender permanentemente de cada participante.

Otro aspecto importante que se debe destacar es que se debe dar a las prácticas la debida importancia desde la formación inicial, partiendo de que el docente tutor tiene la responsabilidad de brindar el acompañamiento necesario y las herramientas necesarias para que el practicante pueda desenvolverse en la comunidad educativa, para que pueda hacer su propia autorreflexión, para que tenga un ambiente de respeto mutuo entre el docente y el aprendiz y las relaciones de colaboración en los espacios pedagógicos.

\section{La insuficiencia de procesos reflexivos en el Eje de Prácticas Profesionales}

Las Prácticas Profesionales deberían estar ligadas a todo el proceso de formación en educación secundaria y superior ya que integran los objetivos de los diferentes ámbitos de 
formación, su marco de referencia se queda sólo en el manejo de habilidades y destrezas, esta condición tiende a perder de vista los vínculos entre los diversos componentes del currículo. La idea predominante tradicionalmente es asumir lineamientos pautados y predeterminados hacia el disciplinamiento de una práctica instrumental, reduciéndola a ser uno de los pocos constructos que se ha mantenido casi inquebrantable frente a la dinámica social en constantes cambios.

Las Prácticas Profesionales tanto como el Eje curricular principal nivel de concreción, podrían constituir el vehículo articulador para la reflexión durante el proceso de formación a fin de fortalecer la relación teoría práctica; ello significa garantizar la posibilidad de reconstruir teoría desde el campo de la acción, lo cual implica la construcción del conocimiento profesional y facilita la adquisición de competencias para consolidar un docente crítico, reflexivo y autónomo. Paradójicamente, estudios realizados por Sayago (2003) y Chacón (2003), demuestran la evidente desconexión entre teoría-práctica en la formación inicial de la carrera Educación Básica Integral, y los Talleres del Eje parecen ámbitos disímiles y sin ninguna relación con las disciplinas.

De lo anterior el autor expresa que no hay conexión con la teoría y la practica en la formación inicial, es por esto que se propone el modelo reflexivo donde el aprendizaje será de formación critica-reflexiva donde el estudiante construya su propio conocimiento. La práctica reflexiva concibe la labor docente más cercana al arte que a la ciencia, valorando que las ciencias aplicada y técnica son necesarias pero insuficientes para esta acción.

Los enfoques socio-crítico y reconstruccionista social, promueven un conocimiento estimulante en los estudiantes, los ayuda a liberarse de sus creencias y valores tradicionales de interpretar el ejercicio de la docencia. De acuerdo con este enfoque las prácticas interpretan la teoría y la práctica como campos mutuamente constituidos y dialécticamente relacionados (Carr, 1996). Las Prácticas llevan implícita una teoría y toda teoría convoca a un modo de hacer y realizar. Campillo (1999. p. 133) refiriéndose a este enfoque, incluye la perspectiva habermasiana, cuando señala que las relaciones establecidas por los estudiantes en período de Prácticas son "eminentemente acciones comunicativas, que los estudiantes realizan mientras piensan, reflexionan, hablan, argumentan, preparan sus discursos, valoran lo aprendido, mejoran y llegan a consenso y todo ello es acción”. En otras palabras, la relación teoría y práctica no actúa separadamente, por el contrario, merece otro ángulo de análisis, se trata de una relación dialéctica práctica, teoría y práctica.

\section{El surgimiento del pensamiento complejo: un nuevo paradigma}

La multidimensionalidad del conocimiento, las demandas sociales y el fenómeno de la globalización reclama mayores esfuerzos al momento de educar, no es suficiente saber, se trata de aprender a aprender en un mundo de constantes desafíos, es comprender a los sujetos, es enseñarlos a elegir aprender. Es asumir lo educativo desde la complejidad, toda vez que el 
desarrollo de un pensamiento de orden superior coadyuve a comprender la globalidad y pluralidad de las sociedades actuales. Es un intento por redimensionar la actitud frente a la organización del conocimiento; esto es, replantearlo desde perspectivas interdisciplinares y transdisciplinares en un afán por dar sentido a la necesidad de formar sujetos con pensamiento crítico y creativo (Morin, 2000).

La propuesta de pensamiento complejo tiene sentido en la formación docente, para considerar el estado de fragmentación del conocimiento en disciplinas fuertemente estructuradas; cuestión determinante en el aislamiento de la teoría y la práctica. Alerta Morín (2000, p. 15), que ese fraccionamiento, "atrofia las posibilidades de comprensión y de reflexión, eliminando también las oportunidades de un juicio correctivo o de visión a largo plazo". Además, Lipman (1997), considera la urgencia de promover el desarrollo del pensamiento complejo como un ente globalizador del pensamiento crítico, reflexivo y rico en recursos metacognitivos o autocorrectivos que sin duda favorecen los procesos de reflexión, y puede contribuir a reinventar las prácticas de enseñanza y orientarlas hacia rutas más humanas y pertinentes con las competencias exigidas a los educadores en la actualidad.

Lo anterior detalla que el pensamiento complejo tiene sentido en la formación docente, donde este promueva el pensamiento crítico-reflexivo donde se desarrolle lo metacognitivo o la autocorrección, donde ayuden al proceso de enseñanza-aprendizaje a orientar a las practicas a que sean más humanas en la actualidad.

En otras palabras, se requiere un docente con pensamiento crítico, promotor de acciones para comprender y cuestionar la realidad y ayudar a transformarla para el bien común, por consiguiente, afirmamos con Mayor, Suengas y González (1995) que el pensamiento crítico es racional, implica las habilidades para reconocer problemas, supuestos, contradicciones, problematiza la propia estructura del pensamiento y está en disposición de contrastarse con otros pensamientos; por tanto, se hace necesario que los estudiantes de Educación adquieran herramientas que les ayuden a desarrollar el pensamiento reflexivo y crítico. Esto indica que puedan comprender la complejidad del aula y el contexto escolar.

Sobre esta base es oportuno preguntarse ¿qué hace la universidad para formar a los futuros educadores desde una perspectiva reflexiva y crítica? Introducirnos en las posibles respuestas, nos conduce a mencionar algunas investigaciones. Diker y Terigi (1997) afirman que la formación inicial está escasamente vinculada con la realidad de los centros escolares por consiguiente los egresados describen dificultades y desconocimiento en relación con aspectos didácticos, relacionados con los contenidos, la planificación, evaluación y la dirección de los grupos de clase. 
Por otro lado, Lee (2005) realizó un estudio sobre el pensamiento reflexivo de los estudiantes de prácticas de matemática en Korea, bajo el paradigma cualitativo, usando la entrevista, el cuestionario abierto y los diarios de los alumnos durante el período de prácticas. La autora concluye que los estudiantes practicantes avanzaron hacia mayores niveles de pensamiento reflexivo durante las prácticas, aun cuando reconoce que lograrlo es una tarea difícil e inacabada. Asimismo, el estudio muestra la influencia de los profesores asesores en las estrategias de enseñanza y en los procesos reflexivos.

De acuerdo con el autor las prácticas tendrán mayor avance cuando se alcance el modelo reflexivo, ya que no es una tarea fácil, pero alcanzable con el trayecto de la puesta en práctica.

Un aporte importante es el de Castellanos (2017) quien afirma que el profesor se convierte en profesional práctico reflexivo en tanto que, significa disposición para percibir la práctica como problemática, identifica situaciones problemáticas en su actuación docente, se distancia de ellas para explicitar y eliminar elementos que le condicionan, y se abre a otras fuentes para interpretar y responder a las mismas. De este modo, consideramos la reflexión como un proceso de pensamiento responsable y sistemático que surge de una situación problemática que requiere disposición para analizar la práctica docente, con el propósito de significar los conocimientos, de comprenderla y actuar ante las situaciones de dicha práctica.

A partir delos aportes de otros estudios atendemos a las categorías que emergen del estudio en donde añadimos algunos rasgos. "Concretamos los rasgos referidos a seis sub -categorías: origen y naturaleza de la situación; definición, dilemas y foco del problema “(Castellanos, 2017).

Las metodologías de estas investigaciones enmarcan que el enfoque cualitativo e interpretativo está orientado a la búsqueda del significado personal de los sucesos; el estudio de las interacciones entre las personas y el entorno; así como los pensamientos, actitudes y percepciones de los participantes.

Por lo tanto los resultados de las investigaciones muestran que los diarios reflexivos, debate, registros de observaciones, técnicas de análisis de contenido, modelo formativo ATLAC podrían mejorar la puesta de la práctica reflexiva.

\section{Los focos de la literatura reciente: evaluaciones, experiencias y análisis de la aproximación de las metodologías}

Cinco documentos describen la reflexión pedagógica en estudiantes de carreras de Ciencias de la Educación, mediante mediciones. El marco conceptual predominante es el de la reflexión crítica, utilizando estrategias más directas como es la investigación cualitativa como la producción escrita, análisis documental y como revisión de producto los diarios reflexivos y portafolios. 
Por ende surge la necesidad de considerar nuevos elementos, factores o variables capaces de impactar el desarrollo reflexivo, que hasta el momento parecen no encontrarse definidos en las comprensiones del fenómeno, como por ejemplo, los de carácter sociopolítico; a la vez que se observa la necesidad de trascender a la investigación sobre experiencias aisladas y de profundizar en investigación orientada a captar elementos críticos para el desarrollo reflexivo y nuevas formas en que la reflexión aproxima la experiencia, se transforman en conocimiento profesional y modifican la práctica.

Tabla 1. Los documentos revisados

\begin{tabular}{|l|l|l|l|l|l|}
\hline \multicolumn{1}{|c|}{ Autor(es) } & Año & \multicolumn{1}{|c|}{ Titulo /Artículo } & Revista & Lugar & Indexación \\
\hline Castellano S. & 2018 & $\begin{array}{l}\text { Sentido otorgado a las situaciones } \\
\text { de la práctica docente: Un estudio } \\
\text { con profesores en formación }\end{array}$ & $\begin{array}{l}\text { Revista } \\
\text { Tecné, } \\
\text { Episteme y } \\
\text { Didaxis. }\end{array}$ & Bogotá & Scielo \\
\hline Olate y Castillo & 2016 & $\begin{array}{l}\text { Desarrollo de procesos reflexivos } \\
\text { desde la percepción de estudiantes } \\
\text { de enfermería }\end{array}$ & $\begin{array}{l}\text { Revista de } \\
\text { psicología }\end{array}$ & Chile & Redalyc \\
\hline Pastell & 2010 & $\begin{array}{l}\text { El aprendizaje reflexivo en la for- } \\
\text { mación inicial del profesorado: un } \\
\text { modelo para enseñar matemáticas }\end{array}$ & $\begin{array}{l}\text { Revista } \\
\text { científica } \\
\text { del caribe }\end{array}$ & México & Redalyc \\
\hline Erazo & 2009 & $\begin{array}{l}\text { Practica reflexiva como estrategia } \\
\text { de desarrollo profesional: presencia } \\
\text { y estructuras en reuniones docentes }\end{array}$ & $\begin{array}{l}\text { Educación } \\
\text { y } \\
\text { educadores }\end{array}$ & Colombia & Redalyc \\
\hline Sayago y Chacón & 2006 & $\begin{array}{l}\text { Las prácticas profesionales en la } \\
\text { formación docente: hacia un nuevo } \\
\text { diario de ruta }\end{array}$ & Educere & Venezuela \\
\hline
\end{tabular}

Fuente: Elaboración propia

\section{CONCLUSIÓN}

Para concluir enfatizamos en la responsabilidad que tenemos quienes formamos docentes y quienes se forman para la docencia crear espacios pedagógicos que favorezcan a ser reflexivos, y es que la reflexión desde las prácticas de formación profesional es esencialmente una forma de ser. Por tanto es necesario la implementación del aprendizaje reflexivo en contextos de innovación de metodologías reflexivas con un enfoque de enseñanza a la práctica de la reflexión donde los docentes sean los facilitadores y guiadores de este proceso. 
Por consiguiente se destaca una percepción positiva de los aprendizajes reflexivos, asumiendo que estos se mantendrán en el tiempo, y asignándole un beneficio integral y amplio, tanto a nivel de la formación profesional, del ejercicio profesional futuro y también a nivel personal.

Mediante estos aportes investigativos fue posible reflejar las áreas de desarrollo del estudiante que desde lo empírico se ocupan a nivel docente y que al explorar en ellas surgen espacios no considerados. Por tanto es relevante alcanzar el desarrollo de los procesos reflexivos y de aprendizaje, como procesos individuales y simultáneos, en que cada uno evoluciona de manera diferente, pero a su vez se influyen mutuamente. De manera similar, el desarrollo de procesos reflexivos genera en los estudiantes una serie de reacciones a nivel del comportamiento, interacciones, pensamientos y emociones, dependiendo de las características individuales del estudiante y del nivel de desarrollo de sus aprendizajes reflexivos.

Por ende surge la necesidad de considerar nuevos elementos, factores o variables capaces de impactar el desarrollo reflexivo, que hasta el momento parecen no encontrarse definidos en las comprensiones del fenómeno, como por ejemplo, los de carácter sociopolítico; a la vez que se observa la necesidad de trascender a la investigación sobre experiencias aisladas y de profundizar en investigación orientada a captar elementos críticos para el desarrollo reflexivo y nuevas formas en que la reflexión aproxima la experiencia, se transforman en conocimiento profesional y modifican la práctica.

"Práctica reflexiva es una modalidad formativa de corte personalista por entender la formación desde la perspectiva de la persona, que parte de su singularidad y por entrelazar profundamente el desarrollo personal con el desarrollo profesional" (Domingo, 2013, p. 386).

\section{REFERENCIAS BIBLIOGRÁFICAS}

Allwright, D. (2005), “Developing principles for practitioner research: The case of exploratory practice", The Modern Language Journal, vol. 89, núm. 5, pp. 353-366.

Alsina, À. (2007), “El aprendizaje reflexivo en la formación permanente del profesorado: un análisis desde la didáctica de la matemática”, Educación Matemática, vol. 19, núm. 1, pp. 99-126.
Brockbank, A. e I. McGill (2002), Aprendizaje reflexivo en la educación superior, Madrid, Morata.

Bruner, Jerome, (1997). La Educación, puerta de la cultura. Madrid, España: Visor Dis, C.A.

Campillo Díaz, M. (1999). El papel del prácticum en la formación de los futuros educadores sociales. En: Revista Pedagogía Social, 35, 129-137 
Carr, W. (1996). Una teoría para la educación. Hacia una investigación educativa crítica. España: Morata.

Carr, W. y Kemis, S. (1988) Teoría crítica de la enseñanza. La investigación-acción en la formación del profesorado. Barcelona: Martínez Roca.

Castellanos, M.T., Flores, P. y Moreno, A. (2017). La reflexión de futuros profesores de matemáticas durante las prácticas de enseñanza. Tesis Doctoral. Universidad de Granada.

Castellanos, M.T., Flores, P. y Moreno, A. (2018). The reflection on practicum: A teaching experiment with Colombian students. Revista Profesorado, 22(1), 413-439.

Chacón, M. (2003) La reflexión y la crítica en los estudiantes de pasantías de la carrera de educación básica integral. Trabajo no publicado para optar al diploma de estudios avanzados (DEA). Universidad Rovira y Virgili- Tarragona: España.

Dewey, J. (1989). Cómo pensamos. Cognición y desarrollo humano. Barcelona, España: Paidós.

Diker, G. y Terigi, F. (1997). La formación de maestros y profesores: hoja de ruta. Buenos Aires: Paidós.

Domingo, A. (2013). Práctica reflexiva para docentes: de la reflexión ocasional a la reflexión metodológica. Saarbrücken, Alemania: Publicia.
Esteve, O. (2004), "Nuevas perspectivas en la formación del profesorado de lenguas: hacia el aprendizaje reflexivo o aprender a través de la práctica”, Actas del ErsteTagungzur Didaktik für Spanisch und Detusch als Fremdsprache, Bremen, Instituto Cervantes

Esteve, O., K. Melief y À. Alsina (2010), Creando $\mathrm{mi}$ profesión. Una propuesta para el desarrollo profesional del profesorado, Barcelona, Octaedro.

Freire, Paulo. (1998). Pedagogía del oprimido. Montevideo, Uruguay: Siglo XXI Editores.

Freud, Sigmund. (1952). Civilization and its Discontents, in The Major Works of Sigmund Freud. Chicago, USA. Great Books of The Western World, Encyclopedia Britannica, Inc.

Korthagen, F. A. (2001), Linking Practice and Theory. The Pedagogy of Realistic Teacher Education, Londres, lea.

Korthagen, f., \& Russell, t. Teachers who teach teachers: reflections on teacher education. Canadian Journal of Education, 1996, 21 (3), pp. 311-358.

Korthagen, FA. Two Modes of Reflection. En: Teacher and Teacher Education, 1993, 9 (3), pp. 317-326. Great Britain: Pergamon Press Ltd.

Lee, Hea-Jin. (2005). Understanding and assessing preservice teachers reflexive thinking. Journal Teaching and of Teacher Education, 21, 699 - 715. 
Lipman, M. (1997) Pensamiento complejo y educación. Madrid: Ediciones La Torre.

Liston, D. y Zeichner, K. (1997). Formación del profesorado y condiciones sociales de la escolarización. $2^{\mathrm{a}}$ ed. Madrid: Morata.

Mayor, J., Suengas, A. y González, J. (1995). Estrategias metacognitivas. Aprender a aprender y aprender a pensar. Madrid: Síntesis.

Morin, E. (1996). Introducción al pensamiento complejo. Barcelona: Gedisa

Morin, E. (2000). El paradigma perdido. Ensayo de bioantropología (6 ${ }^{\mathrm{a}}$ ed.) Barcelona: Kairós.

Pérez, A. (1993). La Interacción teoríapráctica en la formación docente. En: L. Montero y J. Vélez (Editores), Las didácticas específicas en la formación del profesorado. (24-51). Santiago de Compostela: Tórculo.

Perrenoud, P. (2004). Desarrollar la práctica reflexiva. Barcelona: Graó.

Perrenoud, P. “Le Travail sur l'habitus dans la formation des enseignants ana pratiques et prise de conscience". En: PAQUAY, L. et al., Former des EnseignantsProfessionnels: Quelles Stratégies? Quelles Compétences? Belgium: de Boeck \& Larcier, S. A., 1996, pp. 181-207.

Sayago, Z. (2003) El Eje de Prácticas Profesionales en el marco de la formación docente (Un estudio de caso). Tesis de grado de doctora no publicada. Universidad Rovira y Virgili- Tarragona. España.
Schön, D. (1992). La formación de profesionales reflexivos. Hacia un nuevo diseño de la enseñanza y el aprendizaje en las profesiones. Barcelona: Paidós.

Stenhouse, J. (1997). Investigación y desarrollo del currículum. (4ta ed.). Madrid: Morata.

Villar, L. (1999). Enseñanza reflexiva. En L. Villar. (coord.). Un ciclo de enseñanza reflexiva. Estrategia para el diseño curricular. Bilbao: Ediciones Mensajero.

Zabalza, M. (1998). El prácticum en la formación de maestros. En La formación de maestros en los países de la Unión Europea. (322-337). Madrid: Narcea.

Zeichner, K. (1993, diciembre). El maestro como profesional reflexivo. En Cuadernos de Pedagogía, 220, 44-52. 